\title{
IL DIBATTITO AL TEMA DI RITO SLAVO IN POLONIA NELLA LETTERATURA POLACCA NEL PERIODO TRA LE DUE GUERRE*
}

Verso la fine dell'anno 1980 Giovanni Paolo II, il papa degli Slavi, allo scopo di sottolineare i meriti di SS. Cirillo e Metodio nel campo dell'unità europea li dichiarò, accanto al grande Patriarca dell'Occidente San Benedetto, i Patroni dell'Europa. Descrivendo la loro opera missionaria sottolinea che nel raggio della sua influenza si trovavano Bulgaria, Serbia e Russia. E' molto probabile che il detto raggio d'influenza toccò anche la Polonia, e soprattutto la sua parte meridionale (Piccola Polonia). A conferma di tale constatazione viene citato il frammento preso da Vita di Santo Metodio in cui si dice che il Santo aveva invocato il potente „duca dei Vislani” a covertirsi e a farsi battezzare. La leggenda purtroppo non parla del raggio di influenza della missione di San Metodio proveniente dalla Grande Moravia. In Polonia continua, su questo argomento, un animato dibattito nell'ambiente degli studiosi. Alcuni studiosi sostengono che al momento del battesimo ricevuto dal ,potente duca" il cristianesimo di rito slavo in Polonia si era diffuso con maggiore intensità. Riferendosi alle altre notizie storiche coloro asseriscono che San Metodio raggiunse, procedendo nella sua missione, lo Stato dei Vislani e vi fondasse un' autonoma organizzazione diocesana oppure avesse subordinato il paese all'organizzazione ecclesistica, già esistente nella Grande Moravia. Altri studiosi negano, vice versa, al cristianesimo originario dalla Grande Moravia nel sec. IX, la possibilità di sviluppo poichè non trovano nessuna prova che abbia confermato l'esistenza in Polonia del cristianesimo di rito slavo. Lo scopo di questo articolo è l'illustrazione della discussione condotta sulle pagine delle pubblicazioni scientifiche polacche del nostro secolo, specialmente nel ventennio tra le due guerre. L'opera farà riferimento ai testi riguardanti non soltanto la storia della Polonia ma anche la storia dell'arte, l'archeologia, l'architettura, la numismatica, la linguistica, l'onomastica ed le altre, non meno significative discipline.

* L'articolo è un frammento della dissertazione di licenza: Rito slavo sulle terre polacche alla luce della letteratura polacca di ricerca del XX secolo, scritta a. 1995 al Pontificio Istituto di Archeologia Cristiana sotto la direzione del prof. Patrick Saint-Roch.

VOX PATRUM 19 (1999), t. $36-37$ - 30 
Dal momento in cui la Polonia riebbe la sua sovranità, la questione dell'esistenza del rito slavo rianimò di nuovo la letteratura di ricerca di quel periodo. Nel corso delle più varie ricerche riguardanti la storia della Polonia i studiosi affrontavano anche questo problema una volta trattandolo in maniera del tutto marginale un'altra dedicandogli molto spazio. Si profilarono due diversi punti di vista. Gli studiosi ferrati nella materia si divisero in due schieramenti; quello che attribuiva alla missione di Metodio in Polonia un significato piuttosto importante, e quell'altro che rifiutava alla missione il merito di aver avuto un'ampia diffusione e di aver esercitato una significativa influenza in terra polacca.

1. Punto di vista massimalista. Nell'anno 1917 Adolf Szyszko-Bohusz scoprì nel castello di Wawel i resti di una rotonda dedicata a San Felice e Adautto; questo fatto divenne un valido motivo che fece riaccendere il dibattito intorno al tema della penetrazione della missione di Metodio e quindi del rito slavo in territori polacchi e soprattutto in quelli del bacino fluviale dell'alta Vistola. Al vecchio modo di argomentare si aggiunse così la nuova forma - i monumenti materiali, soprattutto quelli archeologici, che come sostenne un appassionato fautore del rito slavo Marian Gumowski, sono un'eccellente fonte storica di gran lunga migliore e più credibile delle leggende degli storiografi o delle ipotesi degli storici ${ }^{1}$. Così quel nuovo reperto divenne agli occhi dei sostenitori del rito slavo una prova dell' esistenza in Polonia del cristianesimo già nel IX secolo.

Ecco le prime conclusioni di questa opinione: l'edificio era sorto nel secolo IX come chiesa intitolata alla Santissima Vergine Maria e sopravvisse al 1241 quando Conrado di Masovia la fortificò. La chiesa, abbandonata e trascurata, venne restaurata per mano di Casimiro il Grande nel 1340. In quella occasione il suo nome venne cambiato e la si intitolò a San Felice e Adautto. Nel 1517 la chiesa venne trasformata in un'abitazione e nel 1806 gravemente danneggiata. Venne riscoperta nel 1917. Per quanto riguarda il nostro argomento, la cosa che più ci interessa è quando e da chi essa era stata eretta. Il suo scopritore A. Szyszko-Bohusz ritiene che la rotonda sia una significativa prova dell'esistenza del rito slavo in Polonia ${ }^{2}$. L'autore ritiene che già cent'anni prima della fondazione del vescovado cracoviense nel 1000 nella zona dell'alta Vistola venisse introdotto il cristianesimo e che la chiesa di San Wenceslao (cattedrale) non fosse affatto la prima costruita sul colle di Wawel ${ }^{3}$.

${ }^{1}$ Cf. M. Gumowski, Szkice numizmatyczno-historyczne z XI w. (Praca Komisji Historycznej Poznańskiego Towarzystwa Przyjaciół Nauk), III, Poznań 1924, p. 80.

2 A. Szyszko - Bohusz, Rotunda świętych Feliksa i Adaukta (Najśw. Maryi Panny), ,Rocznik Krakowski” 19(1918) 51-80 (fotocopia, pp. 2-30).

${ }^{3}$ Ibidem, p. 17. 
Quale sarebbe la prova di ciò? L'analisi della tecnica usata per la costruzione delle mura e delle forme della rotonda sembra confermare che essa esistesse già prima dell'anno 1000. Secondo il parere dell'autore il materiale edile utilizzato per la costruzione della rotonda e il primitivismo delle decorazioni - così differenti dalle decorazioni tipiche delle chiese dell'XI e XII secolo - sono la riprova che essa era stata costruita prima dell'XI secolo ${ }^{4}$. In seguito l'autore si è domandato, quale stile architettonico dominava allora nel cristianesimo e al quale stile si potrebbe attribuire la nostra rotonda? All'epoca in Europa dominavano quel tempo due stili architettonici: lo stile centrale originario dalla Persia e dalla Armenia e lo stile basilicale - tipico per l'Occidente. Nonostante entrambi provenissero dall'Oriente, lo stile basilicale trapiantato in Europa Occidentale vi attecchì profondamente. A questo proposito l'autore dice che nella Polonia dei secc. XI e XII - ,l'epoca in cui l'influenza dell'Occidente era quella vincente, le basiliche venivano erette alla maniera occidentale" . Prima ancora, secondo il parere dell'autore, in Polonia dominavano le influenze orientali originarie dalla Boemia e dalla Moravia dove regnava sovrano il sistema centrale ${ }^{6}$. Gli edifici rotondi, di dimensioni piuttosto ridotte, con le grandi finestre poste in basso, con gli archi semicircolari e modesti decori - così differenti da quelli romanici - potevano essere eretti soltanto dai forestieri giunti dall'Oriente. A questo genere di edifici appartiene la rotonda di Santissima Maria Vergine ${ }^{7}$. L'autore esclude l'influenza dei centri chievani (ruteni) per quanto concerne lo stile della rotonda, perché in Rutenia dominava il puro stile bizantino. Qualora si fosse verificata tale influenza allora la rotonda non sarebbe stata affatto tonda ma viceversa quadrata e avrebbe poggiato su quattro pilastri perché questo era l'aspetto delle prime chiese rutene ${ }^{8}$. L'influenza giungeva quindi dallo Stato della Grande Moravia in cui è stata elaborata una cultura architettonica tipica che non imitava ciecamente le tecniche bizantine.

Prendendo come riferimento una notizia contenuta nella Vita di San Metodio, Szyszko-Bohusz sostiene che nella seconda metà del secolo IX Cracovia, che con molta probabilità era capitale dello Stato dei Vislani, passò a far parte dello Stato della Grande Moravia. Nell'anno 907 l'invasione dei Magiari distrusse questo stato, ma non raggiunse Cracovia dove fino ai tempi del regno di Boleslao l'Intrepido, che incluse la città nello Stato polacco ${ }^{9}$, rimasero vivi i resti della grande tradizione moraviana. Da quel momento l'influenza moraviana cessa, sorgono vescovadi e arcivescovadi subordinati a Roma. Nei secoli

\footnotetext{
${ }^{4}$ Ibidem, p. 20.

${ }^{5}$ Ibidem, p. 22.

${ }^{6}$ Ibidem, p. 23.

7 Ibidem, p. 24.

8 Ibidem, p. 27.

${ }^{9}$ Ibidem, p. 28.
} 
IX e X sui territori della provincia cracoviense (Lo Stato dei Vislani) si scontravano, fino alla vittoria, le influenze di due culture: quella latina con quella greca. La rotonda che fu innalzata in quel periodo porta le tracce dello scontro di due campi d'influenza. E così accanto alla concezione orientale della volta e al sistema tipico dell'arte orientale sorgeva un pezzo di muro che testimoniava l'influenza occidentale ${ }^{10}$. Riassumendo l'autore asseriva che, se la rotonda non fosse sorta come tempio pagano allora con ogni probabilità avrebbe potuto essere costruita come chiesa di rito slavo, e così sarebbe potuta divenire un tangibile segno dell'inclusione dei Vislani nel sistema dello Stato della Grande Moravia. Avrebbe potuto essere costruita nel secolo X e fungere da rifugio ai cristiani moravi dopo l'anno 907. Indipendentemente dall'anno in cui fu costruita (e con ogni certezza si tratta del periodo precedente all'anno mille) la rotonda della Santissima Maria Vergine testimonia in maniera indiscussa che ,il bacino dell'alta Vistola era sito nella zona dell'influenza orientale prima che l'influenza dell'Occidente ebbe la meglio"11.

Il reperto numismatico rinvenuto a Pinczów sito sugli argini del fiume Nida costituisce un argomento interessante, che per di più dovrebbe deporre a favore dell'argomento qui affrontato. Precisamente si tratta di un denaro dell'epoca di Boleslao l'Intrepido con raffigurato sopra il principe e la scritta slava: „Bolesław" e la croce greca sul retro. Risulta interessante la questione della cronologia e il luogo in cui la moneta è stata coniata, che potrebbero aiutarci a spiegare la scritta slava che ci figura sopra. L'autore del testo sul questo denaro Marian Gumowski ${ }^{12}$ ritiene, che la moneta risale ai tempi di Boleslao l'Intrepido ${ }^{13}$. Siccome la moneta era sempre legata con l'organizzazione ecclesiastica ${ }^{14}$ e quindi anche il denaro di Boleslao è stato coniato così come le altre monete dell'Intrepido, nella capitale di una qualche diocesi. Non si poteva trattare della capitale del vescovado latino, poiché si esclude che un sacerdote latino componesse una scritta con le lettere slave. Tale scritta avrebbe potuto essere progettata da un sacerdote slavo e il denaro stesso coniato nella capitale del vescovado slavo ${ }^{15}$. La scrittura del genere potrebbe essere usata da sacerdoti di due riti: quello ruteno (greco-cattolico) e quello slavo, cioè da eredi di San Metodio. L'autore esclude Kiev come probabile luogo in cui era stata

${ }^{10}$ Come sopra, p. 28.

11 Ibidem, p. 23.

12 Cf. M. Gumowski, Szkice numizmatyczno-historyczne z XI w., vol. 2, Poznań 1924, pp. $80-108$

${ }^{13}$ L'autore rifiuta l'ipotesi secondo la quale essa proviene dalla Boemia di cui testimonianza dovrebbe essere la croce patrialcale analoga alla croce raffigurata sui denari boemi di Boleslao II e III. Cf. A. Szelągowski, Najstarsze drogi z Polski na Wschód, Kraków 1909, p. 120.

${ }^{14}$ Nella p. 90 l'autore scrive: ,i reperti numismatici dei sec.X e XI per via delle immagini raffigurate sopra testimoniano una forte influenza della Chiesa anche perché nella magior parte dei casi sono stati coniati nella sede del vescovo".

15 Ibidem, p. 91. 
coniata la moneta ${ }^{16}$, ed in seguito scarta l'ipotesi secondo la quale l'Intrepido avrebbe coniato le monete per le sue terre rutene, perché tali terre non facevano parte dello Stato allora di Boleslao l'Intrepido ${ }^{17}$. Queste monete non sono affatto collegate con l'organizzazione ecclesiastica rutena, poiché essa non esisteva $^{18}$, quindi non resta altro che prendere in considerazione l'unica ipotesi possibile, secondo la quale tali monete sono collegabili con la chiesa occidentale slava approdata in Polonia nel sec. IX insieme ai discepoli di Metodio.

Esprimendo tale giudizio Gumowski cerca contemporaneamente di motivarlo con le argomentazioni già risapute ${ }^{19}$. Egli sostiene che ciascuna delle argomentazioni citate prima, presa singolarmente, possiede un scarso valore probatorio, ma prese tutte insieme confermano in modo piuttosto convincente l'esistenza in Polonia dell'organizzazione ecclesiastica slava ${ }^{20}$. La sua teoria fondata sugli argomenti appena citati si presenta come segue: all'epoca dello Stato della Grande Moravia sulle terre che ne facevano parte, nacque una organizzazione ecclesiastica. Nell'anno 898 su richiesta di Mojmir II (894901) Roma inviò alla volta della Grande Moravia l'arcivescovo Giovanni e i vescovi Danilo e Benedetto. Coloro nominarono l'arcivescovo di Moravia e tre vescovi sufraganei, si ignora di quale rito ${ }^{21}$. Con ogni probabilità uno di

${ }^{16}$ L'autore scrive: ,.... tali monete non sono state rinvenute negli scavi chievani, il conio e il disegno non hanno nulla in comune con quelli usati a Kiev da Vladimiro, Svatopulk o Jaroslaw, (p. 90).

17 Ibidem, p. 90.

${ }^{18}$ Ibidem, p. 94: „L'unica provincia che possedeva l'organizzazione ecclesiastica rutena era quella di Włodzimierz Wołyński, che dopo l'anno 1039 ricevette il vescovo latino".

19 Ibidem, p. 96.

20 Alle dette argomentazioni Gumowski da un'interessante interpretazione. E così il vescovado di Moravia era slavo poiché nell'atto di fondzione del vescovado praghese che risale all'anno 975 il papa Govanni XIII diffida il neo-nominato vescovo dall'uso del rito bulgaro e della linqua slava (Kosmasa Kronika Czechów, Warszawa 1969, p. 143); questo fatto testimonia che il detto rito era molto diffuso in Boemia e che le diocesi confinanti erano di rito slavo:

- la cattedrale di San Felice e Adautto (precedentemente di Santissima Maria Vergine) eretta verso la fine del sec. IX é la superstite del rito slavo. Nella sua pianta e le forme si riscontrano analogie con le chiese morave (p. 99).

- il documento della donazione del Gniezno da parte di Mieszko e Oda che ebbe luogo nell'anno 980 (MPH., I, 148). I confini della della diocesi progettata abbracciano tutto lo stato ad eccezione della Piccola Polonia. La frontiera meridionale arriva fino a Cracovia e non va oltre nonostante che in quell'epoca lo stato polacco abbracciava non solo le terre dei Vislani ma anche quelle della Moravia. La donazione non contempla Cracovia e la Moravia perché esse erano diocesi di rito slavo (p. 100). A favore della slavitá di queste diocesi depone l'antica tradizione del sec. IX, le chiese costruite per favorito l'evoluzione del detto rito (p. 100).

- L'uso ecclesiastico della nomina del vescovo non da canonici del capitolo come di consueto si pracitava nelle diocesi latine ma da tutto il clero della diocesi. L'autore ritiene tale usanza tipica della Chiesa orientale e quindi slavo - metodiana.(p. 103).

21 Ibidem, p. 97; cf. K. Potkański, Kraków przed Piastami (Rozprawa Wydz. Hist. Filozof. Polskiej Akademii Umiejętności, vol. 35), Kraków 1898, p. 81, in cui l'autore sostiene che si trattava di un'organizzazione latina. 
questi vescovi sufraganei potrebbe essere Prochor e il vescovo che succedò a lui uno di nome Prokulf, che come si vede dai nomi, non appartenevano $0^{22}$ alla chiesa latina. Prochor nominato vescovo avrebbe celebrato la funzione $e^{23}$ nella chiesa di Santissima Maria Vergine, mentre Prokulf governava dalla sua sede vescovile di Cracovia per molti anni del sec. X. Dopo l'anno 1000 il rito è stato eliminato e sostituito con il rito latino. La diocesi della Piccola Polonia e quella della Moravia hanno avuto dei vescovi latini. Il vescovo moravo avrebbe dovuto insediarsi a Wrocław unendo in questo modo la Silesia alla Mora$\mathrm{via}^{24}$. Dopo aver suggerito tale ipotesi l'autore ritorna all'analisi relativa al denaro di Boleslao l'Intrepido che dovrebbe essere connesso, come abbiamo già detto, con la chiesa di rito slavo. Siccome il detto rito si sarebbe avallato di una sua organizzazione già prima dell'anno 1000 - di cui la riprova dovrebbe essere, secondo il nostro autore, l'ipotesi sopraccitata - e quindi le monete sono originarie di quel periodo ${ }^{25}$. Rimane da dare la risposta al quesito, dove sono state coniate? Ebbene Gumowski ritiene, che a questo proposito sono da prendere in considerazione due diocesi, quella cracoviense e quella morava. A favore di Cracovia testimoniano sia gli scavi a Pinczów e Wołdawska Niwa, siti lungo il tratto commerciale che da Cracovia andava verso l'Occidente, che il fatto dell'assenza di questa moneta negli scavi moravi e boemi. Lo stile orientale bizantino delle monete é un punto a favore di Cracovia ${ }^{26}$. Sulle monete bizantine l'immagine del monarca e raffigurata en face e lo stesso vale anche per il denaro di cui l'immagine del principe non é rappresentata di profilo come sulle monete occidentali ${ }^{27}$. Inoltre la croce é raffigurata alla maniera bizantina. Qualora la città di Cracovia fosse il posto in cui sono state coniate le monete questo significherebbe che vi erano le influenze bizantine che contagiavano il clero $^{28}$. In questo modo, conclude Gumowski, i denari in quanto il più antico reperto numismatico di Cracovia sono altresi, secondo l'autore, l'argomento a favore dell'esistenza del rito slavo in Polonia. Nell'anno 1000 é stato fondato

${ }^{22}$ Cf. Gumowski, op. cit., p. 99.

${ }^{23}$ L'autore sostiene che la Chiesa di Santa Maria Vergine avrebbe dovuto essere la nostra più antica cattedrale e che come tale serviva a Prochor (p. 169); cf. idem, Problemy wawelskie, „Przegląd Powszechny”, 44(1927) t. 173, p. 66.

${ }^{24}$ Cf. Gumowski, Szkice..., p. 103.

25 M. Gumowski effetua l'analisi delle monete. Stabilisce il loro peso, ch'é pari a 1,07 g ca per ogni denaro e non corrisponde ad altre monete con l'iscrizione Princeps Poloniae che erano in circolazione negli anni immediatamente successivi alla morte di Mieszko I (992) ne tanto meno al denaro coniato in occasione della coronazione di Boleslao l'Intrepido nel 1024. Invece hanno una certa somiglianza, per quanto riguarda il loro peso, con i denari di Boleslao l'Intrepido con l'iscrizione del nome Adelaide, coniati intorno all'anno 1000 . Il loro peso era pari a $1,05 \mathrm{~g}$. Quindi anche le monete slave possono risalire alla stessa epoca. Ciò dovrebbe confermare l'esistenza e l'evoluzione del rito slavo ion Polonia prima dell'anno 1000 (p. 106).

${ }^{26}$ Ibidem, p. 106.

27 Ibidem, p. 107.

${ }^{28}$ Ibidem, p. 107. 
a Cracovia vescovado latino. Tale fatto ha segnato la fine del rito slavo ${ }^{29}$ a Cracovia. L'arcivescovo Aron potrebbe essere quello che insieme al resto del clero di Tyniec abbandonò il vecchio rito e abbracciò quello nuovo, latino, pur mantenendo in vita nella chiesa cracoviense alcune delle antiche usanze ${ }^{30}$. Tutto ciò, sempre secondo lo stesso autore, dovrebbe convincere della presenza della missione di Metodio e Cirillo in Polonia.

2. Punto di vista minimalista. Alle tesi citate sopra si sono opposti numerosi studiosi concordi nel pensare che nulla dimostra l'esistenza e l'evoluzione del rito slavo nella Polonia di primo medioevo. Facendo riferimento al battesimo di Mieszko I, Aleksander Brückner sottolinea che il cristianesimo giunse in Polonia molto prima. Queste irruzioni non erano tuttavia legate all'attivittà dei monaci iroscozzesi oppure a quella dei Fratelli di Salonicco poiché l'idea del cristianesimo penetrava nel territorio degli Slavi prima dell'arrivo della missione di Cirillo e Metodio ${ }^{31}$. Citare le numerose chiese intitolate a San Clemente sparse su tutta la Moravia ha senso soltanto in riferimento alla sua parte meridionale. Simili chiese sorte nella Piccola Polonia non costituiscono alcuna prova inquanto sono state erette grazie alla casata dei Grifiti, e non perché lo vollero i fratelli da Salonicco ${ }^{32}$. Il tempio intitolato a San Felice e Adautto eretto a Cracovia testimonia senza ogni dubbio, che il cristianesimo era ben radicato già nel sec. $\mathrm{X}$, il che non vuole dire altrettanto per quanto riguarda l'influenza del rito slavo ${ }^{33}$.

Secondo il parere di Brückner nel sec. X il rito slavo non avrebbe modo di evolversi, poiché sia Praga che la Boemia da cui il cristianesimo penetrava ampiamente in Polonia, possedevano la gerarchia latina e il rito slavo, ammesso che vi esistesse, era relegato nei posti remoti in cui vegetava appena ${ }^{34}$. Vice versa scorgere nel denaro di Boleslao l'Intrepido le tracce del regno di rito slavo é, secondo l'autore, un fenomeno di „sincera fantasia”, ${ }^{35}$. Concludendo Brückner constata, che in Polonia il cristianesimo era solo di stampo latino e che tale rimase ${ }^{36}$ inoltre che si instaurò a partire dall'anno 966 e non prima.

${ }^{29}$ Molto probabilmente il rito si era ritirato nei conventi. Secondo l'autore il convento di Tyniec, la prima sede della stirpe degli Stasz, che in qualità dei principi vistolani hanno abbraciato come primi questo rito quando San Metodio era ancora in vita, diede analogamente a quello di Sazawa in Boemia, il rifugio al rito slavo. Là il rito era ben protetto e forse, addirittura l'abbazia è stata fondata solo allora quando il rito slavo é stato eliminato dalla cattedrale di Wawel (p. 108).

${ }^{30}$ Cf. Gumowski, Problemy wawelskie, p. 65.

31 Cf. A. Brückner, Dzieje kultury polskiej, vol. 1, Kraków 1931, p. 222.

32 Come lo aveva già sottolineato sia lo stesso K. Potkański (op. cit., p. 195), come anche gli altri.

${ }^{33}$ L'autore sostiene che il detto tempio era stato eretto dopo l'anno 966 (vedi p. 222).

34 Ibidem, p. 222.

35 Ibidem, p. 363.

36 Ibidem, p. 223. 
Nell'anno 1927 il professore Edward Klich ha confrontato circa quaranta termini ecclesiastico - religiosi comuni sia alla lingua polacca che all'antico slavo, che potrebbero costituire l'argomento a favore della ipotesi sull'esistenza del rito slavo nella Polonia dei primordi, a condizione però, che tali terminologie fossero introdotte nelle lingue occidentali slave ${ }^{37}$ dagli stessi Cirillo e Metodio o da loro discepoli. Mentre - constata l'autore - ebbe luogo un processo inverso; le voci citate si insinuarono nell'antico slavo provenendo dalle lingue degli Slavi Occidentali e quest'ultimi le appresero dall'Europa Occidentale. E quindi risulta poco probabile l'attività dei missionari di scuola cirillo-metodiana, che avrebbe influenzato la formazione della terminologia ecclesiastica polacca ${ }^{38}$.

Nell'opera di Tadeusz Silnicki ${ }^{39}$, e precisamente nel capitolo dedicato alla storia della Chiesa in Silesia prima dell'anno 1000, troviamo la reazione alla constatazione secondo la quale in Silesia operavano Santi Cirillo e Metodio oppure loro discepoli. L'autore riconosce, che verso la fine del sec. IX la Silesia avrebbe potuto appartenere all'organizzazione statale e all'organizzazione ecclesiastica della Grande Moravia ${ }^{40}$. Tuttavia manca una qualsiasi traccia dell'operato di Cirillo e Metodio. Mancano inoltre sia la tradizione che le pur minime tracce di rito slavo, e i così detti ricordi di Fratelli di Salonicco sono effetto di una leggenda che nacque in Boemia nei tempi del regno di Carlo IV $(1346-1378)^{41}$.

Tadeusz Lehr - Spławiński ha dedicato molto tempo all'argomento, che é oggetto della nostra discussione. I fautori dell'esistenza del rito slavo hanno trovato nella persona di questo studioso il più convinto critico - oppositore che, con tenacia e in modo alquanto penetrante, metteva in discussione la credibilità delle loro ipotesi. Nel periodo tra le due guerre ha pubblicato sull'argomento

${ }^{37}$ Cf. K. Klich, Polska terminologia chrześcijańska, Poznań 1927, p. 86. L'autore elenca le seguenti voci: anioł (angelo), apostoł (apostolo), archanioł (arcangelo), bałwan (idolo), biskup (vescovo), cerkiew (chiesa ortodossa), chrzcić (battezzare), chrzest (battesimo), chrześcijaństwo (cristianesimo), cmentarz (cimitero), diabeł (diavolo), ewangelia (vangelo), ewangelista (evangelista), jałmużna (elemosina), kanonik (canonico), kielich (calice), kmotr (compare), kolęda (canto di Natale), kościół (chiesa), krzyż (croce), ksiądz (prete), małżonka (consorte), mnich (monaco), msza (messa), ołtarz (altare), opat (abbate), opłatek (ostia), sobota (sabato), szatan (satana), żak (studente), żyd (ebreo).

${ }^{38}$ Ibidem, p. 61. Sulla stessa pagina l'autore constata, che per quanto riguarda l'esistenza del rito slavo sulle terre polacche o per quanto riguarda l'appartenenza del pase dei Vislani all'organizzazione ecclesiastica della Grande Moravia mancano le prove positive.

39 Cf. Historia Śląska od najdawniejszych czasów do roku 1400, Kraków 1939.

${ }^{40}$ L'autore sostiene che ciò si può credere in riferimento al privilegio praghese del 1086 . Anche se si tratta di un falsificato tuttavia il suo autore Kosmas fece riferimento alla tradizione del sec. X e trasmise l'imagine delle relazioni di allora. La metropoli della Grande Moravia avrebbe dovuto estendersi fino agli argini del fiume Bug e Styr e quindi la Silesia insieme alla Piccola Polonia avrebbe avuto i primi contatti con il cristianesimo nell'ambito di questa metropoli (p.9 e ss.).

41 Ibidem, p. 10. 
un articolo intitolato: Missione slava di San Metodio e la Polonia (Misja słowiańska św. Metodego a Polska $)^{42}$ in cui analizza ancora una volta le tesi su cui poggiano le ipotesi dei fautori dell'esistenza del rito slavo nella Polonia all'epoca del primo Medioevo. L'autore sottopone ad una analisi dettagliata i più significativi argomenti dei fautori del rito slavo; come per esempio quel frammento preso dalla Vita di San Metodio riguardante il duca dei Vislani citato nel capitolo I, e constata che coloro traggono da esso le conclusioni troppo azzardate, che non sono risultato dell'analisi della fonte, ma soltanto il risultato di una convinzione /sull'esistenza del rito /assunta a priori/. In realtà - constata l'autore - in questo frammento manca una qualsiasi menzione che potrebbe autorizzare la versione sulla diffusione del cristianesimo con il rito slavo da parte di San Metodio sul territorio della Piccola Polonia ${ }^{43}$. Dopo aver espresso tale parere l'autore cerca di motivarlo in seguente modo:

1. Nel frammento non si parla - come lo vorrebbero i fautori del rito - di un significativo numero di cristiani; 2 . Non si sa se i cristiani rimproverati dal duca erano i suoi sudditi oppure i vicini dei Vislani ${ }^{44}$; 3 . L'invio al duca degli ambasciatori di Metodio sembra poco credibile: Metodio avrebbe piuttosto pronunciato un discorso indirizzato ai suoi sudditi /fedeli/ contenente un avvertimento con il quale avrebbe raggiunto, anche se in modo indiretto, il duca dei Vislani a cui era realmente indirizzato; 4. La prigionia del duca non autorizza affatto - sostiene l'autore - di avanzare l'ipotesi che si trattasse di conquista della Piccola Polonia da parte di Svatopluk e quindi di apertura della porta del paese di Vislani al rito slavo. Con altrettanta probabilità egli potrebbe farsi catturare durante la spedizione in Moravia oppure essere espulso dal proprio paese in seguito alla conquista della Piccola Polonia da parte dei Piast della Grande Polonia $^{45}$. 5. Qualora l'autore di Vita di San Metodio sapesse qualcosa sulla missione di San Metodio nel paese dei Vislani avrebbe senza ogni dubbio cantato le lodi e la gloria dell'eroe della sua opera ${ }^{46}$. E così dalle considerazioni qui citate risulta che la diffusione del cristianesimo nella Piccola Polonia non era, con ogni probabilità, sistematicamente pianificata e che al massimo si potevano constatare i casi sporadici di influenza esercitata da cristiani moravi su Vislani e che quest' ultimi altrettanto sporadicamente, si facevano battezza-

42 „Collectanea Theologica” 13(1932) pp. 2-12; l'articolo é stato pubblicato anche in: Rozprawy iszkice z dziejów kultury Słowian, Warszawa 1954, pp. 182-189. L'articolo é stato pubblicato con alcune modifiche nella rivista bulgaria: „Spisanie na Balgarskata Akademia na naukite” $58(1939)$ p. 73 e ss.

${ }^{43}$ Ibidem, p. 184.

${ }^{44}$ Cf. Wł. Szcześniak, Obrządek słowiański w Polsce pierwotnej, Warszawa 1904, p. 60.

45 A proposito di questa considerazione peraltro già formulata da A. Brückner (cfr. O Piaście, „Kwartalnik Historyczny” $35: 1898$, pp. 313-314), l'autore si dice convinto che essa é altrettanto valida come le altre interpretazioni citate sopra (vedi p. 184).

${ }^{46}$ Cf. Lehr-Spławiński, op. cit., pp. 183-184. 
$\mathrm{re}^{47}$. In seguito l'autore passa all'analisi dei rimanenti argomenti, peraltro a noi già risaputi.

L'autore dedica una scarsa attenzione alle menzioni rutene perché secondo il suo punto di vista esse non meritano di essere prese seriamente in considerazione poiché nascono, sempre secondo il nostro, dalla più pura fantasia ${ }^{48}$. Le fonti latine non forniscono ugualmente alcuna prova significativa. La notizia riportata da Gall in cui egli racconta che la morte di Boleslao l'Intrepido piangevano non solo i polacchi - slavi ma anche i numerosi stranieri, seguaci di rito latino, che si servivano di lingua latina, residenti allora in Polonia. Il frammento della lettera di Matilde si riferisce semplicemente alle lingue conosciute da Mieszko II che era insolitamente colto per quell'epoca. La preghiera ai Santi Cirillo e Metodio del breviario polacco é latina e presa da breviari boemi. I nomi di Prochor e Prokulf sono altrettanto conosciuti in Occidente ${ }^{49}$.

Per quanto riguarda il denaro di Boleslao l'Intrepido, considerato da M. Gumowski argomento chiave a favore della tesi sull'esistenza in Polonia di rito orientale, esso non é più credibile di argomenti precedenti poiché né i fratelli di Salonicco né loro discepoli si servivano della scrittura cirillica ma usavano il più antico alfabeto slavo e cioè l'alfabeto glagolitico $^{50}$. Ne sono prova i testi in antico slavo originari da Moravia e Boemia e scritti esclusivamente con l'alfabeto glagolitico nonché il fatto che tale alfabeto era stato importato da discepoli di Metodio cacciati dalla Moravia e rifugiatisi in Croazia e Dalmazia dove venne mantenuto nei libri liturgici fino ai giorni nostri. Qualora l'iscrizione fosse una traccia della scrittura slava introdotta nella Piccola Polonia da seguaci del rito slavo allora sarebbe stata scritta in alfabeto glagolitico e non in quello cirillico $^{51}$. L'iscrizione sul denaro in cirillico testimonia con ogni certezza, secondo T.L. Spławiński, il legame con la Rutenia poiché là si usava l'alfabeto cirillico preso da bulgari ${ }^{52}$.

Per quanto riguarda l'architettura sacrale l'autore dedica molto spazio ai resti della rotonda di San Felice ed Adautto ${ }^{53}$. Essa non può essere collegata al rito slavo perché é impossibile risalire alla data della sua costruzione e perché il suo stile architettonico si incontra non soltanto nell'Europa orientale ma anche in Italia e nei paesi dell'Europa nord occidentale ${ }^{54}$. La terminologia polacca

${ }^{47}$ Ibidem, p. 185.

48 Ibidem, p. 185.

49 Ibidem, p. 185.

50 Ibidem, p. 186

51 Ibidem, p. 186

52 Ibidem, P. 187.

${ }^{53}$ L'autore segue il ragionamento di K. Potkański e Wl. Abraham e da una valutazione negativa alle chiesette di San Salvatore e di San Clemente intese come prove dell'esistenza di rito slavo.

54 Ibidem, p. 188, cfr. Wł. Abraham, Recenzja pracy Szyszko-Bohusza („Kwartalnik Historyczny" 34:1920, pp. 116-118), in cui l'autore indica le analogie italiane (Battisteri a Biella e 
inerente al culto cristiano non testimonia affatto l'antico legame tra la Polonia e il rito orientale poiché molte voci sono state prese sia da dialetti germanici che da quelli di Slavi occidentali ${ }^{55}$.

Infine contro la tesi sull'esistenza di rito slavo in Polonia si schiera il fatto che i Frattelli di Salonicco hanno dedicato molte forze alla lotta contro le mire tutt'altro che amichevoli del clero tedesco. San Metodio nella sua veste di arcivescovo era l'oggetto degli attacchi di Svatopluk fortemente influenzato da vescovi latini ed anche da vescovi tedeschi. Inoltre era perennemente accusato di diffondere il rito slavo. Se questo fosse lo stato di cose che regnava nello stato della Grande Moravia allora ci viene un dubbio sulla probabilità della diffusione del rito slavo al di fuori dei confini della Grande Moravia ${ }^{56}$. „E allora - conclude prof. Spławiński - nulla ci autorizza a ritenere che l'attività di San Metodio abbracciò la Polonia" e che vi é stato innestato il rito slavo ${ }^{57}$. Le riflessioni di Tadeusz Lehr - Spławiński sono state considerate „un bellissimo epitaffio" per la tesi dell'esistenza di rito slavo in Polonia ${ }^{58}$. I successivi sforzi degli studiosi che miravano ad un ulteriore approfondimento di questo indubbiamente rilevante aspetto della storia dei primordi della nostra nazione era stata interrotta dallo scoppio della seconda guerra mondiale. La ricerca riprese il suo corso solo alla fine della guerra.

\section{DEBATA NA TEMAT RYTU SŁOWIAŃSKIEGO W POLSCE W POLSKIEJ LITERATURZE MIĘDZYWOJENNEJ}

(Streszczenie)

Artykuł ukazuje przebieg dyskusji w polskiej literaturze naukowej XX-lecia międzywojennego, dotyczącej istnienia obrządku słowiańskiego w Polsce wczesnośredniowiecznej, odnośnie którego zaznaczyła się polaryzacja stanowisk wśród uczonych. Zwolennicy jego istnienia, do argumentów znanych dotychczas nauce, dodają nowe, zaczerpnięte $\mathrm{z}$ badań archeologicznych, numizmatycznych i lingwistycznych; na ich podstawie próbują uzasadniać, iż chrześcijaństwo nie tylko dotarło do Polski już w IX wieku wprost z Wielkich Moraw, gdzie działali święci Cyryl i Metody, ale

a Galliano) e le analogie dell'Europea nord occidentale (Rotonda di St. Léonard in Haute Vienne e St.Hubert nelle Ardenne).

${ }_{55}$ Ibidem, p. 188.

56 Ibidem, p. 189.

57 Ibidem, p. 189.

${ }^{58}$ Cf. Jerzy Zathey, O kilku przepadtych zabytkach rękopiśmiennych Biblioteki Narodowej w Warszawie, in: Studia z dziejów kultury polskiej, Warszawa 1949, pp. 1-32. 
zapuścić ono miało tak głębokie korzenie, że zorganizowane zostało w struktury kościelnej organizacji. Przeciwnicy z kolei, wykazując kruchość tych hipotez, nie zaprzeczają samej możliwości docierania chrześcijaństwa do Polski już w IX wieku od strony państwa Wielkomorawskiego; odmawiają mu jednak większego wpływu i znaczenia, gdyż brakuje na to, w ich przekonaniu, poparcia zarówno w źródłach pisanych jak i archeologicznych. Dyskusja jednak w nauce polskiej wokół tego problemu nadal trwa. 